\title{
Chinese specialist consensus on imaging diagnosis of intracranial arterial dissection
}

\author{
Yan-Min Wang ${ }^{1}$, Yi-Sen Zhang ${ }^{2}$, Bin-Bin Sui ${ }^{3}$, Xin-Jian Yang ${ }^{2 *}$, Pei-Yi Gao ${ }^{3 *}$, Neurointerventional Expert Committee \\ of Chinese Congress of Neurological Surgeons, Chinese Interventional Neuroradiology Society of Chinese Stroke \\ Association and Young Physicians Committee of Chinese Congress of Neurological Surgeons
}

\begin{abstract}
Intracranial arterial dissection is an important cause of stroke in young and middle-aged adults, but there are no domestic or globally-accepted criteria for the diagnosis and classification of intracranial arterial dissections. The evidence base for the benefits of diagnostic and interventional angiography techniques is lacking, and the imaging features of intracranial arterial dissections are not yet universally recognized or understood by clinicians. These issues influence the diagnosis and treatment of intracranial arterial dissection. Therefore, a consensus conference was convened by Neurointerventional Expert Committee of Chinese Congress of Neurological Surgeons, Chinese Interventional Neuroradiology Society of Chinese Stroke Association and Young Physicians Committee of Chinese Congress of Neurological Surgeons. The aim of this document is to report an expert consensus on the imaging diagnosis of intracranial arterial dissection. The participating clinical experts utilized their judgment and experience. Every effort was made to achieve consensus among the committee members. The expert consensus was intended to provide guidance, but they are not to be considered prescriptive for any individual patient and cannot replace the judgment of a clinician.
\end{abstract}

Keywords: Intracranial arterial dissection, Expert consensus, Imaging diagnosis

\section{Background}

An intracranial arterial dissection (IAD) occurs when circulating blood penetrates the intracranial vessel wall through a tear in the intima, and extends through and between the tissue layers of the wall. This results in hematoma or spontaneous intramural hematoma formation, causing stenosis, occlusion or disruption of the vessel [1]. If dilation occurs, an IAD is defined as an intracranial dissecting aneurysm (IDA) [1]. The etiology of IAD is still unclear, but epidemiologic studies have identified arterial hypertension, oral contraceptive use, migraine, a history of trauma and recent infection as risk factors [2, 3], and an elevated risk of IAD may also be inherited [2]. IAD is rare. While its precise incidence is not known, IAD is reportedly more common in Asians than Europeans and

\footnotetext{
* Correspondence: yangxinjian@bjttyy.org; cjr.gaopeiyi@vip.163.com 2Department of Interventional Neuroradiology, Beijing Neurosurgical Institute and Beijing Tian Tan Hospital, Capital Medical University, Beijing 100050, China

${ }^{3}$ Department of Neuroimaging, Beijing Tiantan Hospital, Capital Medical University, Beijing 100050, China

Full list of author information is available at the end of the article
}

Americans, and the posterior circulation is more frequently affected than the anterior circulation (accounting for $76 \%-93 \%$ of lesions) [4]. Despite its rarity, IAD is an important cause of stroke in young and middle-aged adults, and may be manifested as headache, an ischemic event, subarachnoid hemorrhage (SAH) or mass effect $[2$, $3,5]$. IADs are increasingly identified with non-invasive imaging modalities [6], but there are no domestic or globally-accepted criteria for the diagnosis and classification of IADs. The evidence base for the benefits of diagnostic and interventional angiography techniques is lacking, and the imaging features of IADs are not yet universally recognized or understood by clinicians. These issues influence the diagnosis and treatment of IAD.

\section{Diagnostic imaging strategy for intracranial arterial dissection}

IADs can cause headache, ischemia or hemorrhage, or act as a space-occupying lesion due to progressive enlarged of the dissected artery. Early recognition and accurate diagnosis of IAD informs the choice of clinical 
management strategies, and may thus reduce the risk of stroke [4]. The diagnosis and classification of IAD relies heavily on imaging techniques, especially magnetic resonance imaging (MRI), digital subtraction angiography (DSA) and computed tomography angiography (CTA).

\section{Imaging characteristics of intracranial arterial dissection}

The diagnosis of IAD requires a comprehensive assessment of its location and characteristics, and its influence on adjacent structures. Characteristic imaging features of IAD include: (1) eccentric luminal narrowing with increased external vessel diameter; (2) the 'pearl-and-string' sign or segmental stenosis; (3) intramural hematoma (IMH); (4) the double-lumen sign; (5) presence of an intimal flap; and (6) a dissecting aneurysm. An intimal flap and the double-lumen sign are pathognomonic of IAD. The appearance of these features may be different depending on the imaging modality used. If the imaging features are typical, the diagnosis of IAD can be made by CTA, magnetic resonance angiography (MRA) or DSA alone. Lesions with atypical features may require investigation with more than one imaging method. Comprehensive assessments of images of the lumen and vessel wall are needed for clinicians to understand the underlying pathophysiology, guide treatment and make accurate judgments about prognosis.

(1)The presence of an intimal flap (Fig. 1) is pathognomonic of IAD; it is manifested as a linear, hyperintense signal separate from the vessel lumen on black-blood MRI. Han et al. have reported that $\mathrm{T} 2$-weighted imaging (T2WI) is capable of detecting approximately $50 \%$ of intimal flaps, and less frequently intimal displacement, findings that suggest the technique is superior to DSA [7]. The vessel wall and intraluminal flap are better visualized with gadolinium enhancement (Fig. 1b) [8]. Three-dimensional (3D) reconstruction of black-blood sequences allows an intimal flap to be viewed at any angle. If the presence of an intimal flap is unclear, a dissecting lesion must be differentiated from a thrombosed aneurysm. The differential diagnosis of the lesion should be considered on the basis of its location, shape, and how it changes over time.

(2) The double-lumen sign (Figs. 1 and 2) is also pathognomonic of IAD [4]. The true vessel lumen appears round, but may be deformed or narrowed; there may also be incomplete occlusion and bypass of blood flow into the pseudolumen. The signal intensity of the true lumen is hypointense on black-blood MRI sequences and hyperintense on bright sequences. The pseudolumen is frequently crescentic, containing blood that flows between intimal layers over a potentially extending distance. The pseudolumen may contain hematoma. Heterogeneous signal within the pseudolumen indicates the presence of slow and turbulent flow.

(3) Intramural hematoma (Figs. 3 and 4) is typical in IAD, but MR imaging findings are highly dependent on the age of the IMH, the appearance of surrounding structures and the sequence used. An IMH can often be directly visualized on MRI, accompanied by increased wall thickness with a smooth rim. The MRI signal intensity varies according to the age of the IMH due to changes in its paramagnetic iron components. An IMH with a methemoglobin component is more likely to be detected in the subacute and early chronic stages due to its crescentic hyperintense appearance, accompanied by eccentric reduction in the diameter of the arterial lumen. New heavily T1-weighted imaging (T1WI) techniques, such as magnetization-prepared

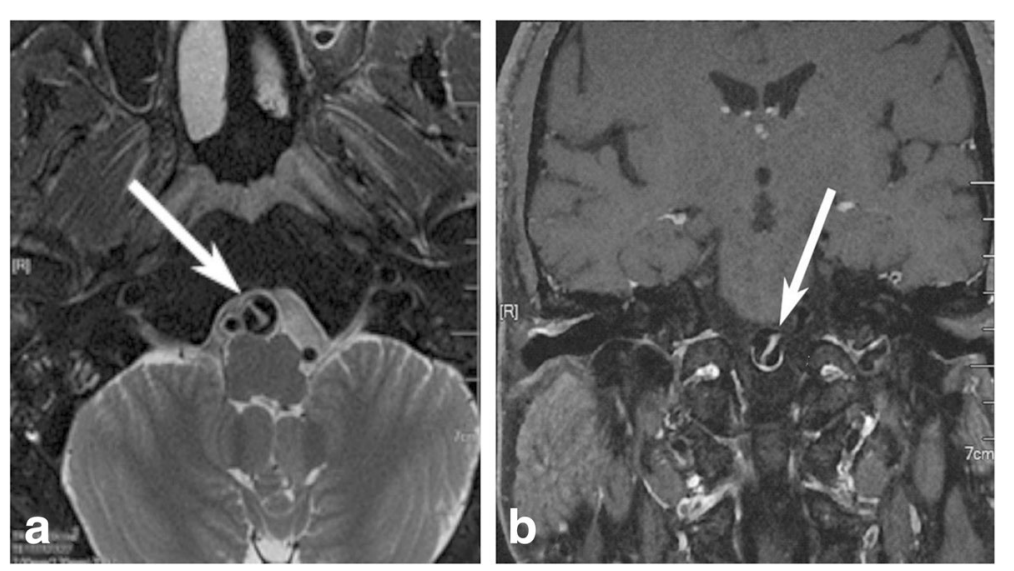

Fig. 1 Magnetic resonance images showing an intimal flap in a left vertebral artery dissection. a A T2-weighted image showing a dilated left vertebral artery, with an increased external diameter and hyperintense signal caused by a linear intimal flap (arrow) in the vessel lumen. b A contrast-enhanced 3D black-blood T1-weighted image showing significant enhancement of the intimal flap (arrow) and the wall of the left vertebral artery 


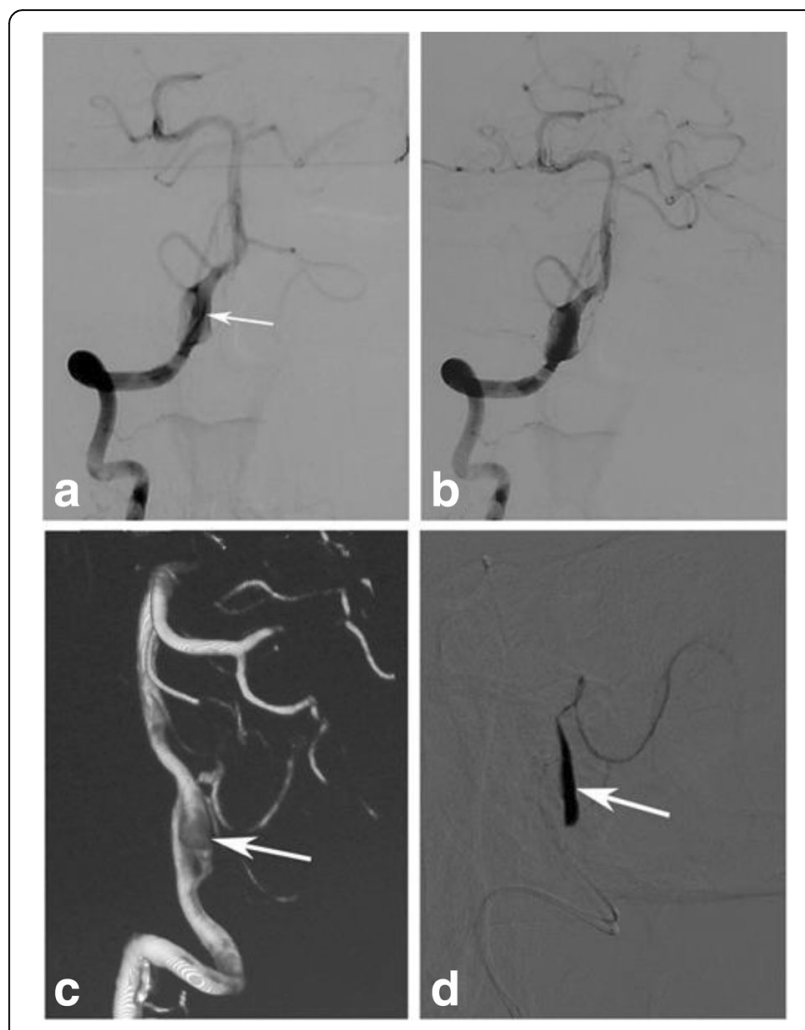

Fig. 2 Double-lumen sign on digital subtraction angiography. a Inflow of contrast agent in the lumen of the $\mathrm{V} 4$ segment of the right vertebral artery is separated by an intimal flap (arrow), resulting in the doublelumen view. $\mathbf{b}$ The whole dissecting aneurysm is filled by contrast agent in the late arterial phase. c 3D rotational angiography outlines the external contour, divided into the true lumen and the pseudolumen (arrow). $\mathbf{d}$ Selective angiography after a microcatheter tip has been advanced just into the pseudolumen show the pseudolumen and posterior inferior cerebellar artery branching from the distal part of the aneurysm (arrow), confirming the existence of two lumens

rapid gradient echo (MP-RAGE) or simultaneous non-contrast angiography and intraplaque hemorrhage (SNAP), can detect hemorrhage as a hyperintense signal against a gray-scale background (Fig. 3c). Nevertheless, MRI has limited capability to detect an IMH in the acute ( $<7 \mathrm{~d}$ ) and chronic ( $>2$ months) stages, as the IMH is isointense on T1WI and the boundary of surrounding structures will likely be unclear. On sensitivity-weighted imaging (SWI) of intracranial vertebral arterial dissection, the presentation of IMH closely corresponds to that of dissection, and SWI reportedly has a sensitivity of $90.0 \%$ and specificity of $96.6 \%$ for the detection of IMH [9]. An IMH usually becomes isointense or undetectable within 6 months [10]; its hyperintense signal intensity may, however, be visible for longer, although it is not clear whether such an appearance is associated with an elevated risk of recurrent hemorrhage [11]. It is important to differentiate IMH from intraplaque hemorrhage on the basis of its location, the proportion of the vessel wall involved and the total increase in vessel wall thickness.

(4) Luminal changes (Figs. 4 and 5) caused by arterial dissection include dilation with stenosis, irregular stenosis with dilation, and complete occlusion. Changes in the dissecting lumen can be clearly identified with time of flight MRA (TOF-MRA), contrast-enhanced MRA (CE-MRA) and highresolution MRI (HR-MRI). Irregular dilation of the affected segment can be seen in a dissecting aneurysm. Large dissecting aneurysms are frequently accompanied by obvious displacement of surrounding tissues due to compression. Recurrent bleeding with thrombus formation inside the aneurysm exhibits 'onion skin' mixed signal intensity on MRI (Fig. 4) [12]. The mass effect of a vertebrobasilar IDA may distort the brainstem in some cases. Focal cerebral ischemia in the vascular territory of the affected vessel and subsequent changes of other brain tissues may also be seen on MRI.

Dissection of the anterior circulation is less common. Angiography often shows segmental stenosis, but may fail to detect signs of intimal flap, dissecting aneurysm or a double lumen, [13] and CTA and conventional MRA are not reliable means of making the diagnosis. Vessel wall imaging with HR-MRI may lead to the diagnosis of middle cerebral artery (MCA) dissection. Lee and colleagues have reported that it could demonstrate an intimal flap and a tapered pseudolumen with intraluminal hematoma in a case of MCA dissection [14].

\section{Magnetic resonance imaging for the diagnosis of intracranial arterial dissection}

Plain MRI and 3D TOF-MRA are conventionally used to diagnose dissection; according to a 2009 systematic review of 21 articles published from 1992 to 2008, MR techniques have a specificity of $29 \%-100 \%$ and a sensitivity of $50 \%-100 \%$ [15]. Test characteristics for MR techniques such as MRI and MRA are relatively similar to those for CTA for the diagnosis of carotid and vertebral artery dissection [15]. Computed tomography/CTA is subjectively favored for vertebral dissection, as more features of cervical artery dissection can reportedly be seen than conventional MRI or MRA when multidetector CT is used [16]. Although MRI is thought to be more sensitive than other techniques for the detection of subacute IMH, the hyperintense IMH signal may be mistaken for flow-related signal in TOF-MRA. A combination of black-blood sequences and CE-MRA can help to distinguish blood flow from an adjacent IMH. A pitfall of TOF-MRA is that signal loss caused by laminar blood flow or blocking artifacts may lead to flow-related 

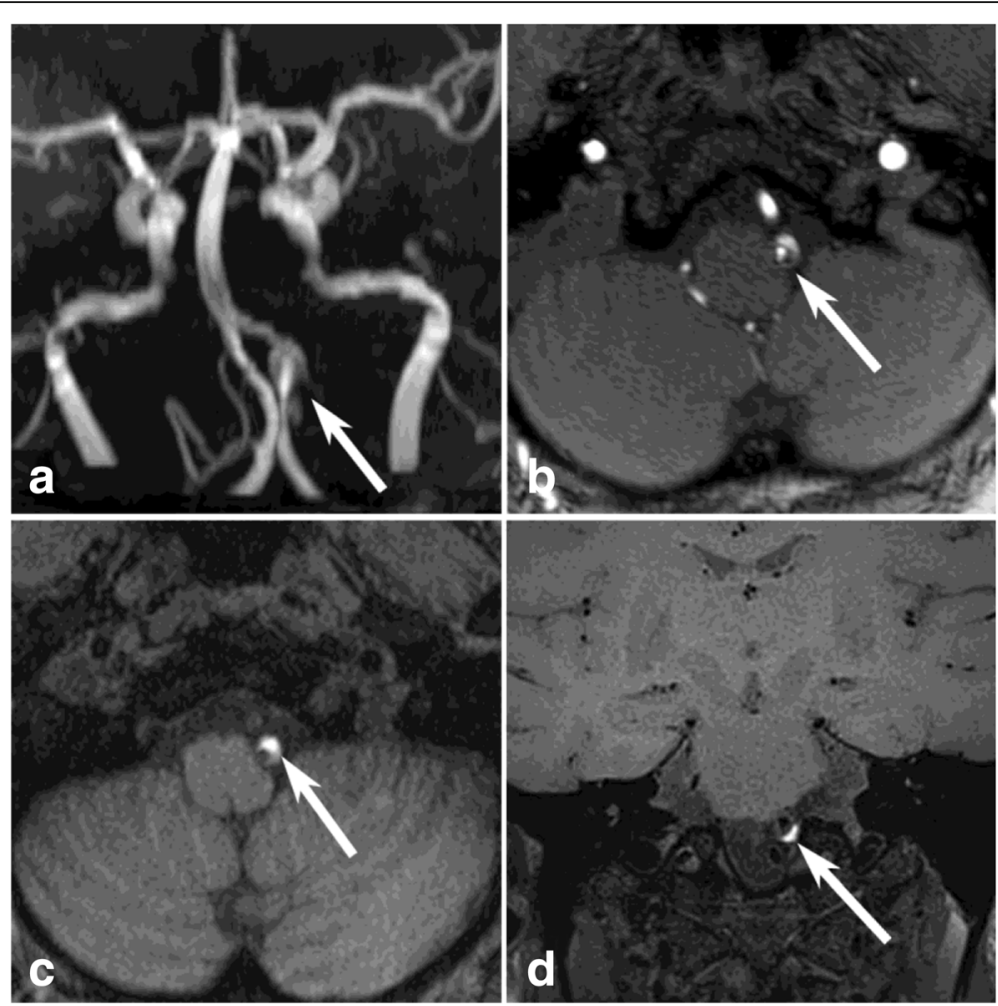

Fig. 3 Imaging of a dissecting aneurysm of the left vertebral artery. (a) 3D time of flight (TOF) magnetic resonance angiography shows focal dilation of the left vertebral artery with hyperintense signal (arrow). (b) Source TOF data show the left vertebral artery separated by a linear hypointense signal into a right posterior true lumen and a left anterior pseudolumen (arrow). (c) Magnetization-prepared rapid gradient echo (MP-RAGE) sequence showing an intramural hematoma (arrow) with a bright hyperintense signal in the pseudolumen. (d) 3D black-blood T1weighted imaging showing an intramural hematoma (arrow) with a bright hyperintense signal

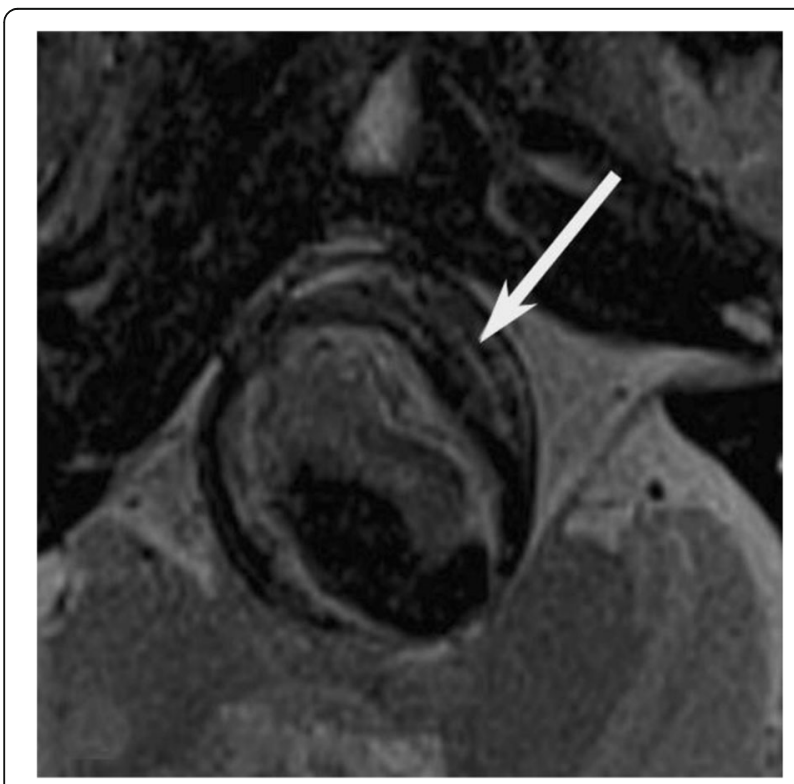

Fig. 4 Imaging of a dissection involving the inferior segment of the basilar artery. T2-weighted imaging shows substantial dilation of the basilar artery with 'onion skin' characteristics (arrow) owing to multiple layers of dissection/thrombus in the vessel wall

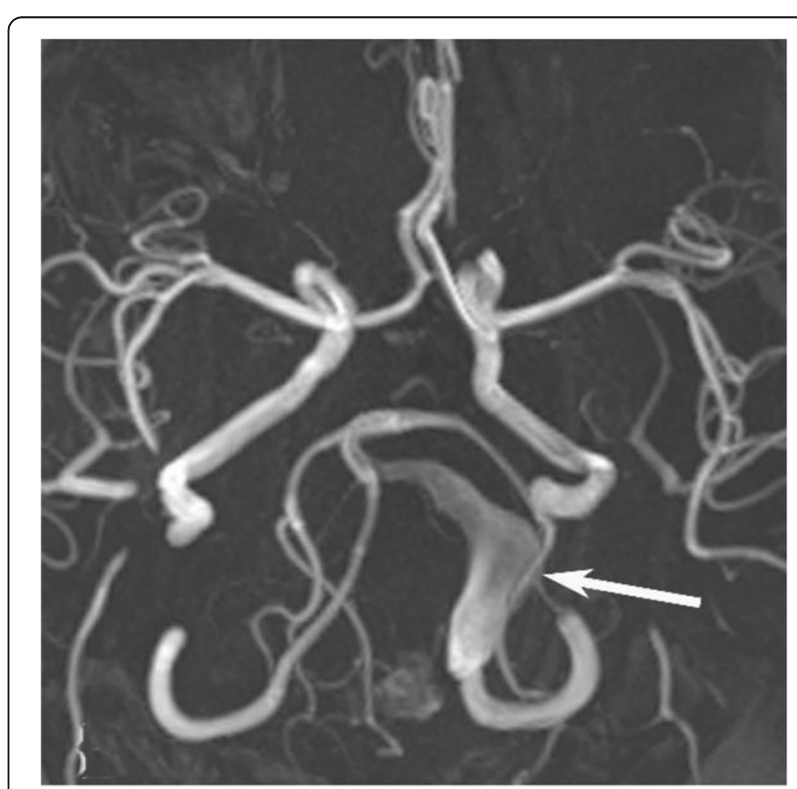

Fig. 5 3D time of flight magnetic resonance angiography showing a dissecting aneurysm (arrow) of the left vertebral artery 
signal attenuation in some horizontal-running arteries. Compared with CT, MRI is superior for the visualization of posterior fossa lesions and for the assessment of vertebrobasilar dissections.

Vessel wall HR-MRI is increasingly used in clinical practice. The technique has been extensively used for carotid artery imaging, highlighting its potential diagnostic utility for intracranial arterial lesions. The technique reportedly offers excellent visualization of the intracranial arterial wall [7, 17], and is an effective non-invasive means of diagnosing IADs [18-21]. Nonetheless, the evidence base is still somewhat lacking, and consequently HR-MRI has not been widely adopted in clinical practice.

Black-blood sequences acquired with HR-MRI can be used to obtain two-dimensional (2D) T1-, T2- and proton density weighted images (PDWI) with thin-slice scanning perpendicular to the vessel. High-resolution 3D TOFMRA should be included in the assessment of the vertebrobasilar arteries. As the sensitivity of heavily T1WI for the detection of hemorrhage is high, it may be more reliable for the detection of IMH $[22,23]$. Development of $3 \mathrm{D}$ sequences expands the examination range of HR-MRI, allowing a variety of aspects and angles for clinicians to observe lesions [24-26]. Three-dimensional black-blood T1WI can identify IMH more accurately than conventional spin echo and TOF-MRA imaging [27]. In contrastenhanced HR-MRI, enhancement of the vessel wall is a useful means of detecting an intimal flap [17].

\section{Digital subtraction angiography for the diagnosis of intracranial arterial dissection}

Digital subtraction angiography is the conventional imaging modality. It is an accurate means of assessing the shape of the affected artery, providing important hemodynamic information and allowing collateral circulation to be documented [4]. Three-dimensional rotational angiography (3D-RA) provides high spatial resolution of reconstructed $3 \mathrm{D}$ images so that the characteristics of an artery can be assessed in cross-section [28]. Findings pathognomonic of arterial dissection (the double-lumen and/or 'pearl-and-string' sign) are observed more frequently with 3D-RA (90\%) than conventional DSA. The main pitfall of DSA is that it does not allow visualization of the arterial wall, and diagnosis of IADs with normal-looking lumens can be challenging. If the lumen is stenotic or occluded, an IAD may be difficult to distinguish from atherosclerosis, arteritis or vasculitis. Furthermore, DSA alone cannot provide the complete view of an IAD, as it cannot detect any associated IMH or a partially thrombosed aneurysmal lumen.

Patients with spontaneous IAD usually also have arteriosclerotic lesions and atheromatous plaque. Fibromuscular dysplasia or abnormal collagen protein content can lead to weakness of the arterial wall in some patients
[3]. Thus, DSA examination should be standardized to avoid procedure-related complications such as embolism and vascular injury. Dynamic images of the whole circulation period should be examined carefully. In lesions with unclassifiable lumen shapes, X-ray exposure time should be prolonged to the venous sinus phase, which may also detect contrast agent retention. In spontaneous vertebrobasilar dissection, $62 \%-76 \%$ of IADs reexamined by DSA resolve [29, 30], although a minority of cases may progress rapidly with substantial changes in lesion characteristics observed over days or even hours [31, 32]. Given the potential speed of these changes, it is critical to compare images with previous ones to illuminate dynamic changes in the status of lesions. Although DSA was traditionally the first-line investigation for IAD, it has largely been supplanted by CTA and MRA, but it still has a role in patients with SAH or acute ischemic stroke, as it allows endovascular treatment to be undertaken.

The radiologic findings of DSA in IAD are as follows: (1) irregular or segmental stenosis; (2) tapered occlusion; (3) 'pearl-and-string' sign or string sign (irregular fusiform or aneurysmal dilation with or without proximal and/or distal stenosis; Figs. 6 and 7); (4) contrast agent retention in the pseudolumen (Fig. 8); and (5) the

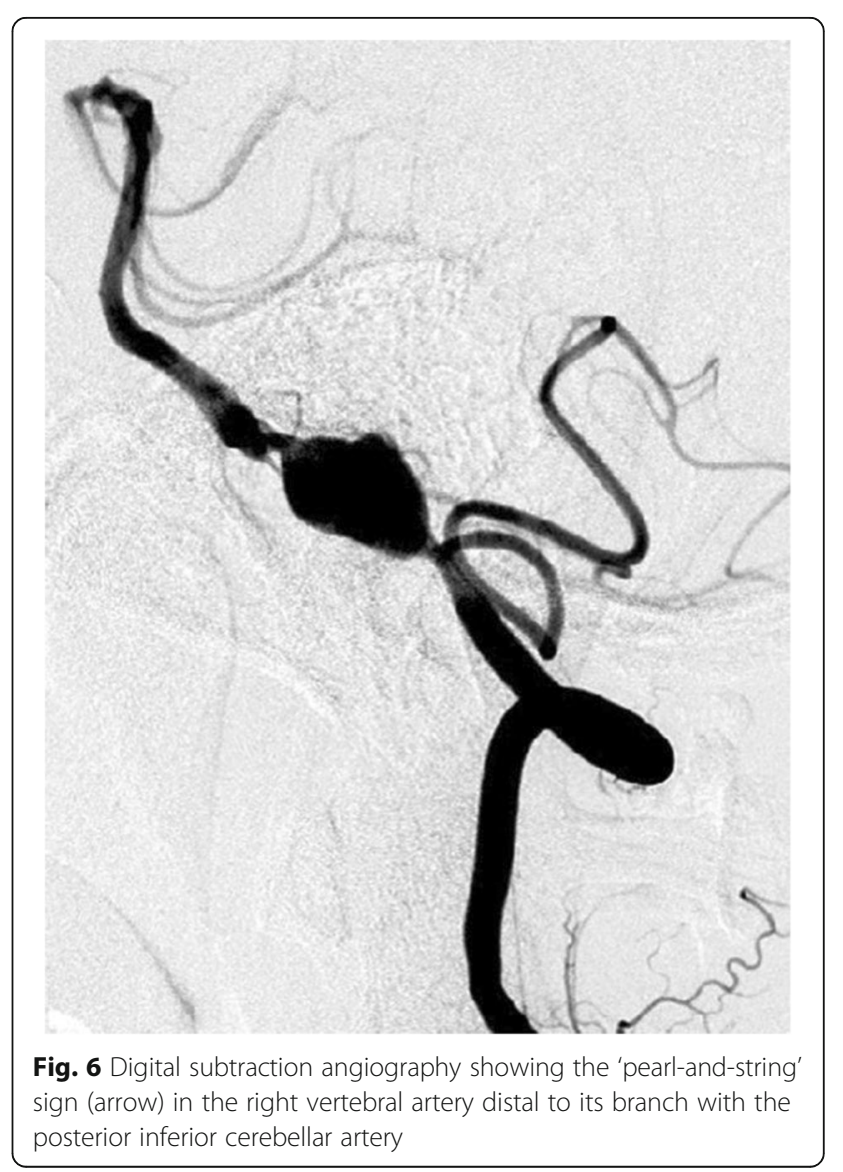




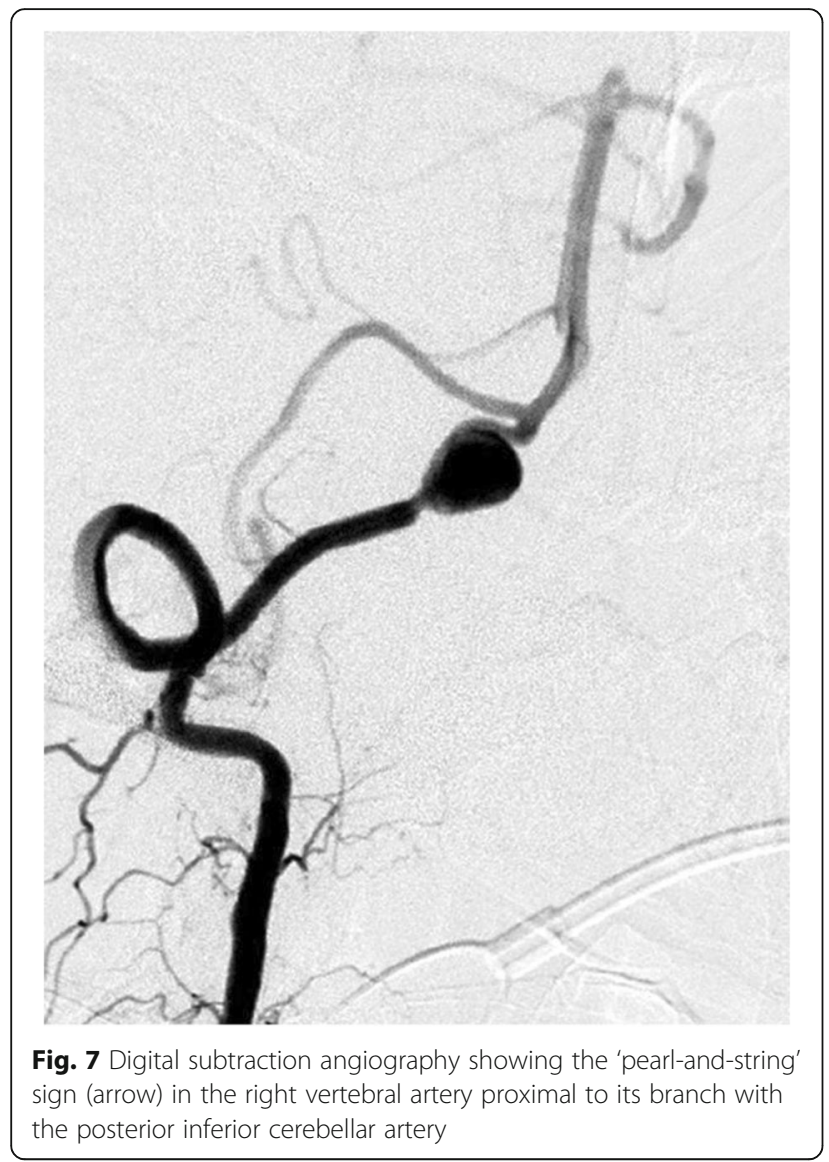

double-lumen sign (Fig. 2). The 'pearl-and-string' sign and segmental stenosis are the most common findings [33]. An intimal flap or a double lumen, which are pathognomonic of dissection, are detected by DSA in fewer than $10 \%$ of cases [34]. Nonetheless, DSA can identify faster blood inflow in the true lumen, contrast agent retention in the pseudolumen and intraluminal flap-like structures. The angiographic appearance of aneurysmal

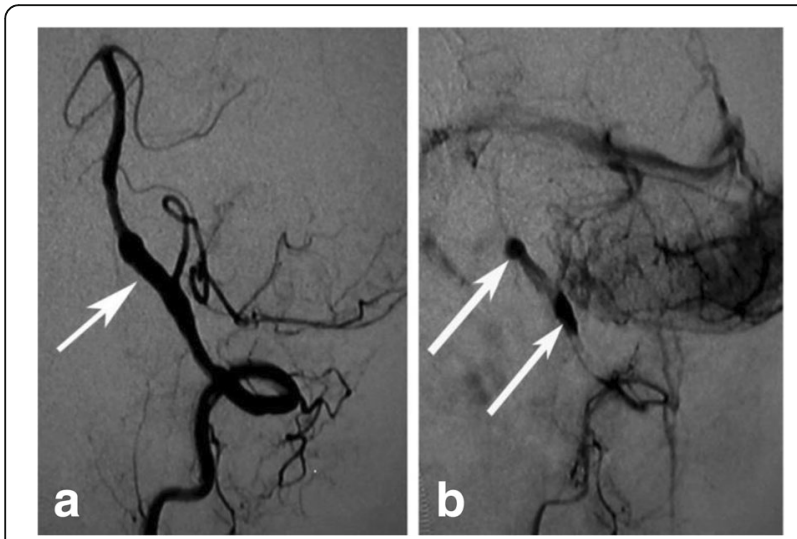

Fig. 8 Contrast agent filling phase of digital subtraction angiography. a Contrast agent filling the aneurysmal lumen in the arterial phase (arrow). b Retention of contrast agent in the venous phase (arrow) dilation with stenosis is more frequent in $\mathrm{SAH}$, and it is more common to see stenosis without dilation in patients with infarcts, and fusiform or aneurysmal dilation in unruptured cases without infarcts.

\section{Computed tomography angiography for the diagnosis of intracranial arterial dissection}

Multisection CTA is a sensitive and accurate means of diagnosing IAD $[35,36]$. Compared with DSA, the sensitivity, specificity and accuracy of multisection CTA for the diagnosis of extracranial vertebral dissection are $100 \%, 98 \%$, and $98.5 \%$, respectively [37]. Multisection $\mathrm{CT}$ volume scanning examination, with a fast scanning speed and wide range, can provide high-resolution images of the arterial lumen and wall, and evaluate both extracranial and intracranial segments. The detection rate and diagnostic accuracy of craniocervical dissections have improved as CT technology has advanced [38]. Post-processing of CTA source data can increase the rate of detection of small lesions by providing global, multi-angle reconstructions. Thin axial CTA source data is very useful for the identification of specific signs of vascular disease, helping to identify intimal flaps, but skull base and tooth artifacts can impair CT images and lead to misdiagnosis. Radiation doses and administration of iodine-containing contrast agents may also cause adverse reactions and increase the risk of complications during IAD detection. The conduct of image acquisition in CTA also allows non-enhanced cross-sectional CT images of the head to be obtained, for the assessment of any associated ischemic or hemorrhagic changes. Computed tomography also has the advantage of rapid image acquisition of vascular and cerebral tissue, bone and extracranial organs with minimal movement artifact. Consequently, CTA is the preferred imaging technique for traumatic dissections.

Detection of eccentric luminal narrowing with increased external diameter on CT is highly suspicious of IAD, and the crescent abnormality on cross-sectional images is suggestive of IMH. Source CTA images can present a likely IMH due to accompanying luminal narrowing and increased external diameter; an IMH is usually isodense and does not enhance after contrast administration. In their 2009 study, Lum and colleagues reported that the ratio of lumen diameter to total diameter is a sensitive means of detecting IAD [39]. For an IAD with a normal luminal appearance, caution must be exercised when mural thickening is detected on source CTA images. Increased total vessel diameter and vessel wall thickening can be found in $93.3 \%$ and $97.7 \%$ of dissected vertebral arteries, respectively, but they can also be seen in other vascular diseases [40]. If a doublelumen sign is observed, a diagnosis of dissection can be made with confidence. The true lumen is round or oval, 
and an incompletely occluded lumen has rapid inflow and contrast agent filling, while the hypodense crescentic pseudolumen exhibits slow flow velocity and contrast agent retardation due to extension of the arterial wall. Detection of an intimal flap or a dissecting aneurysm is a reliable sign of dissection. An intimal flap is less frequently seen than an IMH, requiring careful observation on thin source CTA data (Figs. 9 and 10).

\section{Imaging differential diagnosis for intracranial arterial dissections}

To make a diagnosis of IAD, it is essential to distinguish a range of pathologic conditions affecting cerebral vasculature. While anatomic diagnosis of arterial narrowing is made with reasonable accuracy, ascribing the etiology for the stenosis remains challenging in clinical practice as radiographic mimics of IAD such as intracranial atherosclerosis, intracranial vasospasm (reversible cerebral vasospasm syndrome, RCVS), fibromuscular dysplasia and to a lesser extent arteritis/vasculitis are often encountered [41]. In addition, IDAs with intramural hemotoma formation should be distinguished from thrombosed intracranial saccular aneurysms. Thus, multimodal imaging techniques are needed to offer the potential information to discriminate between these pathologies. Existing modalities for imaging the lumen of blood vessels, including CTA, TOF MRA or DSA may have a limited ability to differentiate vascular pathologies. Looking beyond the arterial lumen, direct imaging of the blood vessel wall offers further insight into the pathogenesis.

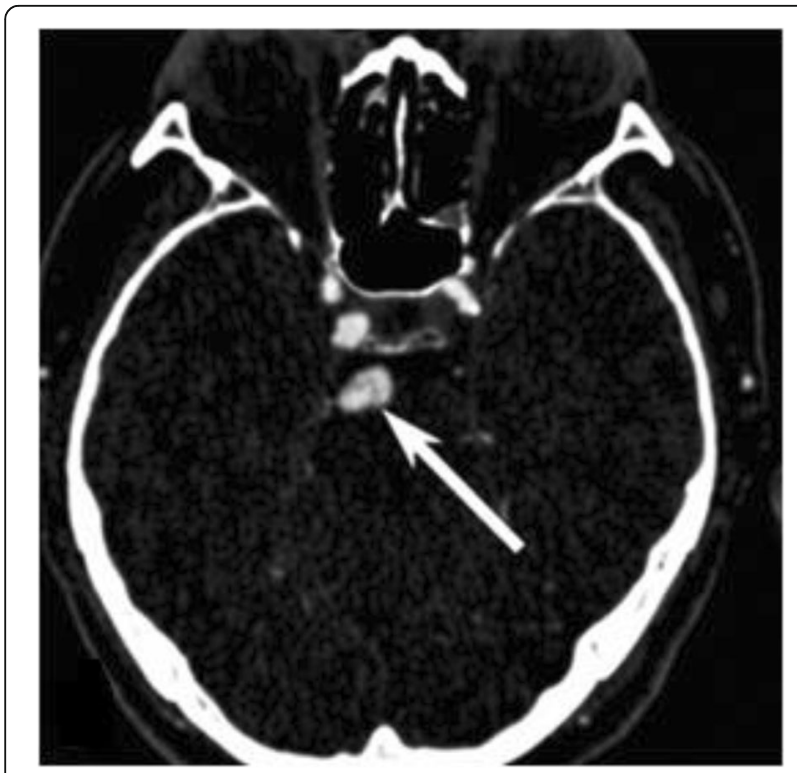

Fig. 9 Basilar dissecting aneurysm on source image of computed tomography angiography. There is irregular luminal thickening of the basilar artery, and the lumen is divided by an irregular isodense linear intimal flap (arrow)

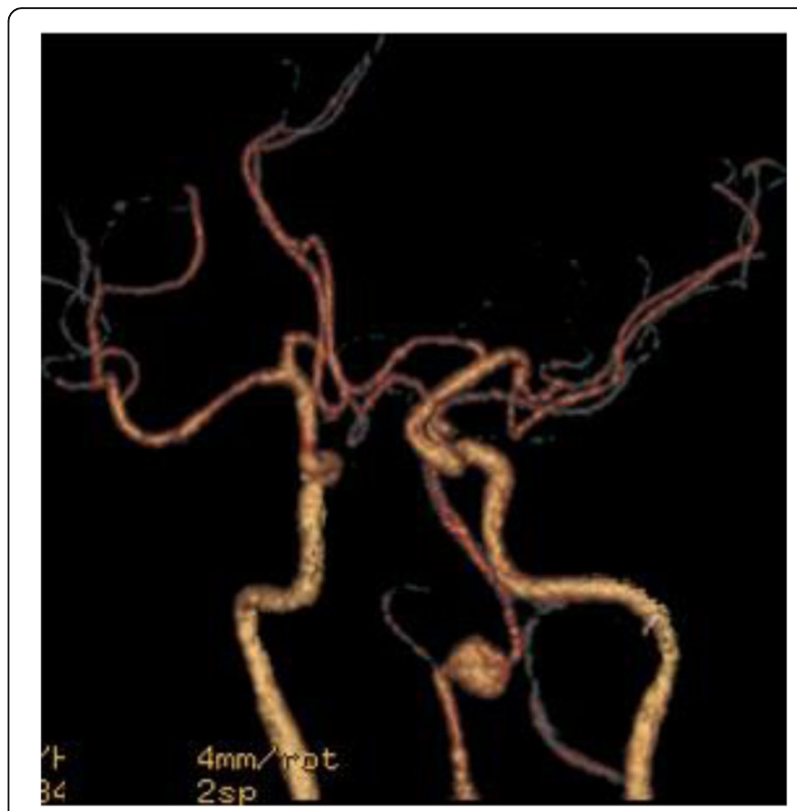

Fig. 10 Right vertebral artery dissecting aneurysm (arrow). Volume rendering of computed tomography angiography showing increased vessel diameter of the right vertebral artery with focal aneurysmal dilation

As we know, intracranial atherosclerosis has been suggested to be the most common cause of ischemic stroke worldwide, accounting for $33-50 \%$ of all strokes in China [42]. We need to be careful to avoid misreading irregular stenosis of intracranial atherosclerosis with pearl-and-string sign or tapered stenosis of dissection Most atherosclerotic segments display some compensatory enlargement (positive remodeling [PR]) when the stenosis degree is less than $30-40 \%$, but it may be inadequate to prevent luminal stenosis (inadequate PR) [43]. Some vessels may paradoxically shrink at the lesion site (negative remodeling [NR]), exacerbating rather than compensating for luminal loss [44]. Characterization of atherosclerotic plaque using high resolution MRI is already well established [45-47].

Cross-sectional and longitudinal imaging by HR-MRI at $3 \mathrm{~T}$ are recommended to be performed to depict intimal and adventitial vessel wall boundaries in the diseased vessel segment for differentiating IMH from intraluminal thrombus [48]. Basi-parallel anatomic scanning (BPAS)-MR imaging, which allows visualization of the external contour and surface appearance of the vertebrobasilar artery, can also be added to differentiate arterial dissection from atherosclerosis or hypoplasia [49].

On vessel wall HR-MR imaging, intracranial atherosclerosis generally reveals eccentric thickening with irregular surface, with variable signal intensity within plaque due to different components. Intracranial dissection can also present as eccentric wall thickening with enlargement of outerwall diameter. If the "double- 


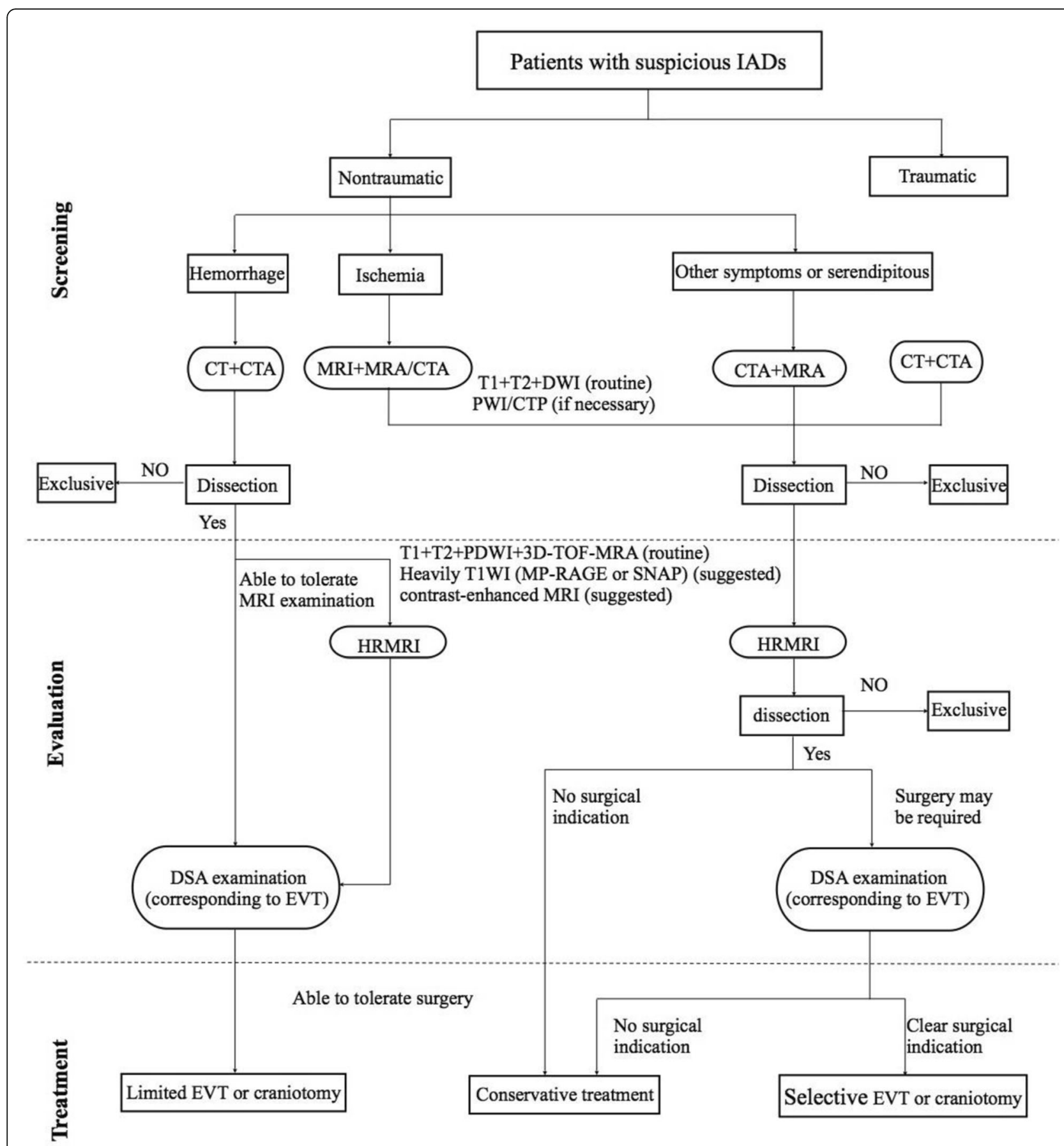

Fig. 11 Protocol for diagnosis of intracranial arterial dissection. Abbreviations: DWI, diffusion weighted imaging; PWI, perfusion weighted imaging; PDWI, proton density weighted imaging; MP-RAGE, magnetization-prepared rapid gradient echo; SNAP, simultaneous non-contrast angiography and intra-plaque hemorrhage; HR, high-resolution. EVT, endovascular treatment

lumen" [17] or the intimal flap was visualized, the diagnosis of IAD can be confirmed. Bright wall elements on non-enhanced T1 sequence is another feature of IAD, indicating methemoglobin in the arterial wall. However, this bright signal can also be found with intraplaque hemorrhage. Involvement of long segment along the longitudinal axis of vessel wall could be a very important sign of dissection different from other vascular diseases. Unfortunately, it is not common for intracranial dissections.

Patterns and intensity of postgadolinium enhancement of vascular lesions on MRI T1 post-contrast vessel wall imaging can be used to make differential diagnosis. Intracranial atherosclerotic plaques usually display 
variable enhancement; IAD may demonstrate a similar enhancement pattern to atherosclerosis. However, contrast imaging can increase the visualization of intimal flap, which can help the differential diagnosis. No enhancement of IMH associated with acute dissection is distinguishable from clear enhancement of intraluminal thrombus $[18,50]$. Focal enhancement of IMHs after gadolinium application may be seen possibly due to neovascularization or the vasa vasorum sprouting into the IMH.

Vasculitis often shows characteristic concentric and smooth wall thickening, with intense and homogeneous enhancement; reversible cerebral vasoconstriction syndrome (RCVS) has minimal to no enhancement and minimal wall thickening [48]. If diagnosis of dissection is still uncertain, it would be necessary to make short-term follow-up by HR-MRI to gain information with regard to recanalization, degree of stenosis, formation of pseudoaneurysm, and appearance of new dissections [18].

\section{Recommendations}

\section{Imaging strategy for intracranial arterial dissection}

(1) Intracranial imaging should be undertaken in all patients with typical clinical symptoms (hemorrhage, ischemia and neurologic dysfunction) and those with a history of head or neck trauma, especially young and middle-aged patients. (2) Computed tomography with CTA, or MRI with MRA, are recommended for initial screening. (3) Cerebral DSA is an important tool for diagnosis and allows endovascular treatment to be undertaken. For a confirmed or highly suspicious IAD, DSA is recommended to achieve accurate evaluation of the shape of the lumen, hemodynamic status, and the collateral circulation of the dissecting artery. (4) Computed tomography angiography is recommended for suspected traumatic artery dissection or emergencies. In the event of images being inconclusive or not diagnostic of IAD, HR-MRI or DSA should be performed. (5) A CT scan should be performed in patients with suspected SAH to identify the bleeding site and severity. (6) In cases with suspected cerebral ischemia, MRI and diffusion weighted imaging are recommended to establish whether there is acute infarction. If necessary, cranial CT perfusion or perfusion weighted imaging is recommended to identify areas of ischemia-reperfusion. (7) Conventional TOF-MRA or contrast-enhanced MRA can be used to screen for and locate vessel lesions; HRMRI should be focused on the affected artery when IAD is likely, and include 2D black-blood T1WI, T2WI and PDWI perpendicular to the artery. We recommend that 3D TOF-MRA should be included for examination of the vertebrobasilar artery. One heavily $\mathrm{T} 1$ weighted sequence, such as MP-RAGE or SNAP, is also recommended, as they are very sensitive means of detecting the signal intensity of an IMH. At least one 3D sequence is also recommended to obtain different views of lesions. (8) If IAD is confirmed or highly suspicious on DSA, HRMRI should be used as a complementary method to reveal the pathologic condition of the vessel wall, and establish the presence of an IMH, intimal flap and/or mass effect. Additional plain cerebral CT or MRI is helpful to document associated intracranial hemorrhagic or ischemic events. (9) As some IADs may progress rapidly, images should be viewed in the context of previous findings, if available. Short-term imaging follow-up is suggested for dynamic observation of cases in whom diagnosis is uncertain (Fig. 11).

\section{Imaging diagnosis of intracranial arterial dissection}

(1) The diagnosis of IAD can be made on DSA if there is tapered stenosis or occlusion, segmental stenosis with dilation (the 'pearl-and-string' sign) or contrast agent retention in the late venous phase. (2) A definitive diagnosis can be made if a double lumen and/or an intimal flap are detected by CTA, MRA OR DSA. The double-lumen sign is manifested as two lumens (that enhance with contrast to different extents) separated by a linear isodense structure on CTA, a hypointense signal in the true lumen with mixed signal intensity in the pseudolumen caused by turbulent flow on black-blood HR-MRI sequences, and retention of contrast agent in the pseudolumen on DSA. An intimal flap can be easy to observe on T2WI and contrastenhanced black-blood HR-MRI as a linear or septal structure located within the vessel lumen. (3) A subacute IMH exhibits hyperintense signal on T1WI, TOF and MPRAGE sequences; detection of an IMH supports the diagnosis of IAD. (4) Either TOF-MRA, CE-MRA, CTA or DSA can be used to assess luminal changes in the affected artery, such as irregular narrowing, dilation, stenosis with dilation or complete occlusion (Fig. 12).

\section{Imaging classification strategies for intracranial dissecting aneurysm \\ Background}

The IDA is a subtype of IAD. As for IAD, there are no globally or nationally recognized standards for the diagnosis and classification of IDA. Non-atherosclerotic cerebral fusiform and dissecting aneurysms have been classified by Mizutani et al. [51] into four types: classic dissecting aneurysms, segmental ectasia, dolichoectatic dissecting aneurysms, and saccular aneurysms unrelated to the branching zones. Basilar trunk aneurysms have been categorized by Saliou et al. [52] also into four types: acute dissecting aneurysms, segmental fusiform ectasia, chronic mural bleeding ectasia, and saccular aneurysms. Both taxonomies have been recognized by some scholars, but neither was designed specifically to classify dissecting aneurysms. The clinical manifestations, pathophysiology, treatment and outcomes of saccular and 


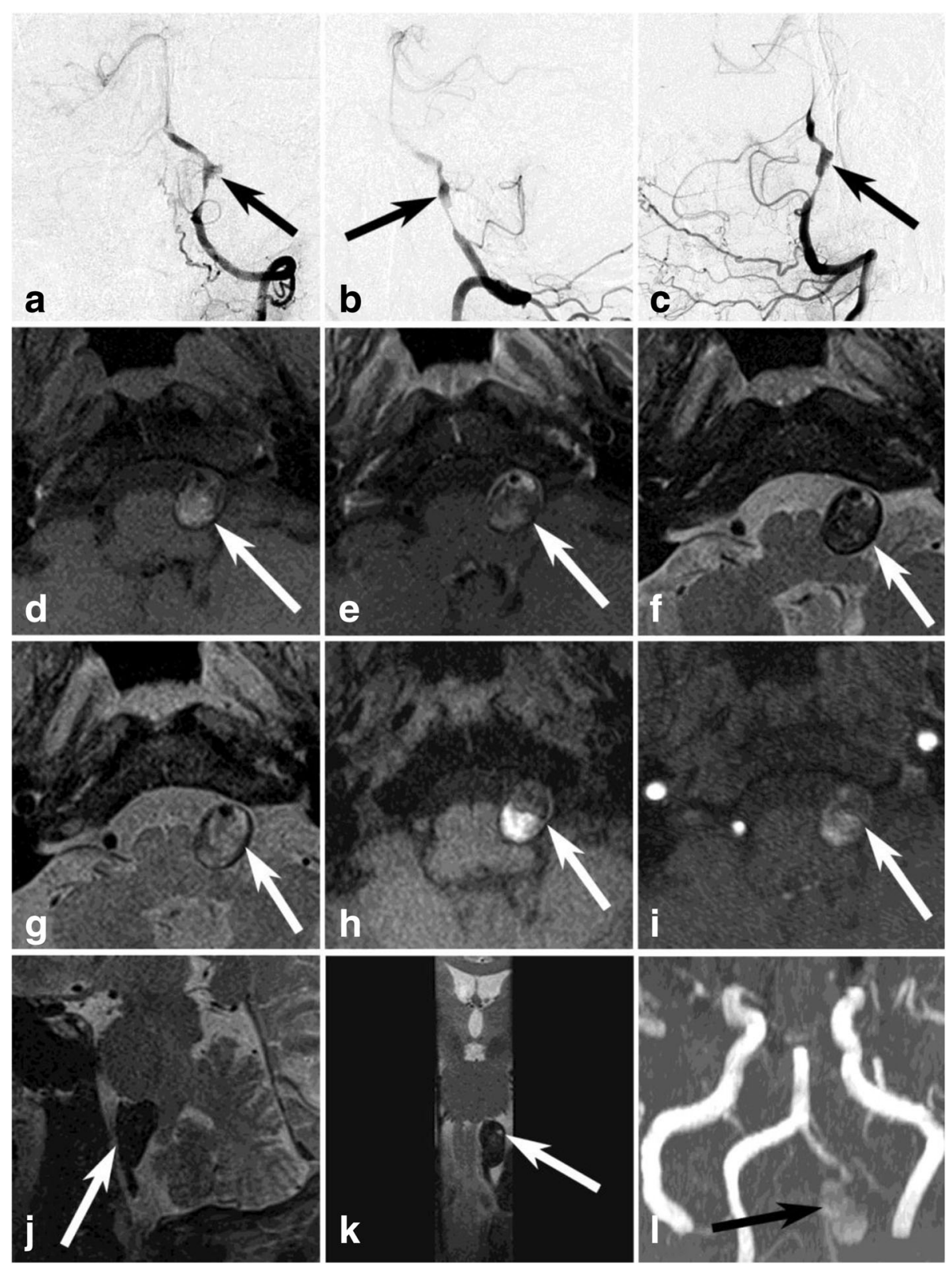

Fig. 12 Images of a dissecting aneurysm. a-c Frontal, lateral and left anterior view demonstrate the 'pearl-and-string' sign (arrow) in the left vertebral artery. $\mathbf{d}$-I Lesion (arrow) viewed by different high-resolution magnetic resonance image sequences and from different viewing angles. d-i Axial T1-weighted images (Wl), contrast-enhanced T1Wl, T2Wl, perfusion weighted imaging, magnetization-prepared rapid gradient echo and 3D time of flight. j Saggital T2WI. $\mathbf{k}$ Coronal T2WI. I Magnetic resonance angiography

dissecting aneurysms differ, so it is important to have a precise means of classifying the morphologic characteristics of IDAs $[2,20]$. In China, an IDA may be referred to as a 'a fusiform aneurysm', 'a serpentine aneurysm,' 'a pseudoaneurysm,' 'a thrombosed aneurysm' and 'vertebrobasilar dolichoectasia'; however, there is no clear taxonomy of these concepts. Some terms refer only to dissecting aneurysms, while others may include nonaneurysmal lesions. The ideal classification should be clearly defined and comprehensive, to inform appropriate clinical-decision making and guide treatment strategies.

\section{Recommendations}

Based on previous international morphologic classifications, $[51,52]$ and in the context of differences in the pathophysiologic and morphologic characteristics of the vessel lumen and wall, and differences in treatment strategy and clinical prognosis, we recommend that intracranial fusiform and 


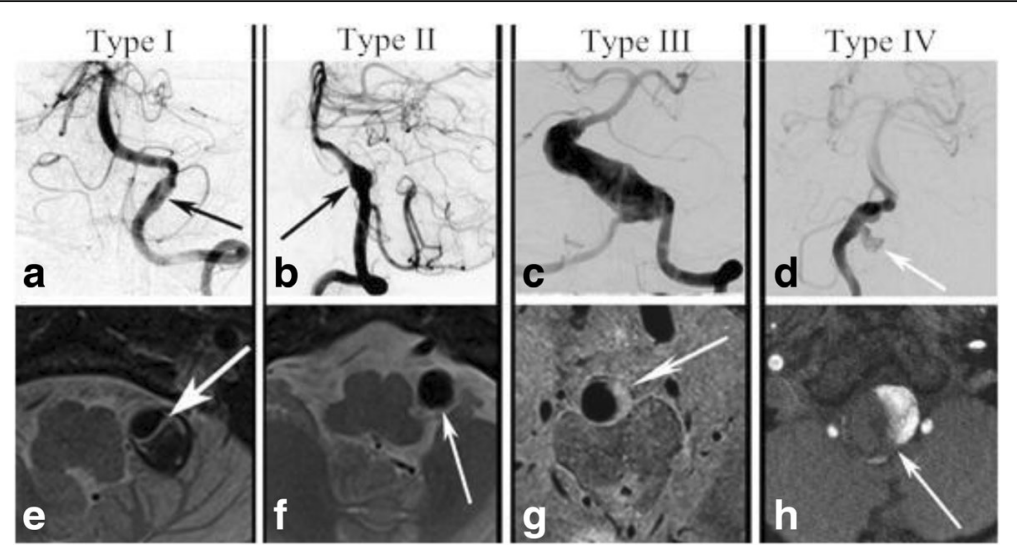

Fig. 13 Proposed classifications of intracranial dissecting aneurysms (arrow). a-d Digital subtraction angiography images of the classical type (Type I), segmental dilation (Type II), dolichoectasia type (Type III) and large mural bleeding ectasia (Type IV). e-h Corresponding magnetic resonance images of each type

dissecting aneurysms are categorized into four types: classical type, segmental ectasia, dolichoectatic type, and large mural bleeding ectasia (Fig. 13) [53].

(1) Classical type (type I) presents as an irregular fusiform or aneurysmal dilation, accompanied by proximal and/or distal stenosis (the 'pearl-and-string' sign) on DSA, CTA and MRA. Typical intimal flaps and IMHs ( $<10 \mathrm{~mm}$ in diameter) can often be found on HR-MRI. Pathologic findings consist of local disruption of the internal elastic lamina, formation of intimal breakthrough, communication with the arterial lumen, intimal and medial tears, flow of blood through the tear, and the presence of a pseudolumen packed with mural thrombosis. This type is thought to be an early manifestation of dissection. Endovascular treatment by stenting or stent-assisted coiling is considered the most appropriate treatment to achieve the best outcomes.

(2) Segmental ectasia (type II) presents with segmental dilation, without proximal or distal stenosis, without evidence of an intimal flap or the double-lumen sign, and without obvious hematoma in the aneurysmal wall. Typical pathologic findings of this type are segmental ectasia owing to degeneration of the internal elastic lamina or atrophy of medial smooth muscle, often accompanied by collagen deficiency and endothelial proliferation. Typical lesions are detected without concomitant atheromatous deposits, but with a smooth luminal intimal surface, no thrombus formation, a thin wall and larger size. This type of aneurysm can be treated conservatively, as they are considered stable and in the chronic repair stage.

(3) Dolichoectatic type (type III). Dolichoectatic dissecting aneurysms are most frequently located on the basilar artery. They are tortuous, fusiform and of giant size, with extensive involvement of the longitudinal axis of the vessel with a thickened intima, intimal flap and IMH. Pathologic findings are most often a result of fragmentation of the internal elastic lamina (IEL) and multiple dissections of the thickened intima, with thrombus formation across the disrupted intima. In contrast with large mural bleeding ectasia (type IV), dolichoectatic aneurysms may affect the artery along its entire length, but local IMH is not that extensive. Despite these findings, treatment is nonetheless conservative due to the high risk of complications with intervention; there is no clear evidence to support surgery for this type of aneurysm.

(4) Large mural bleeding ectasia (type IV) are characterized by obvious IMH, frequently with multiple layers of recurrent hematoma, presenting as a crescent or 'onion-skin' appearance on MRI. The diameter of this type of aneurysm may exceed $25 \mathrm{~mm}$, resulting in substantial brain compression. The shape of the lumen is variable, and may appear normal on DSA in some cases. Pathologic characteristics of this type are wide disruption of the internal elastic lamina, intimal hyperplasia, multiple layers of thrombus formation and neovascularization within the thickened intima. Any mass effect can be addressed by craniotomy, at which the lesion can be excised and vascular bypass performed if necessary, but operative risks are nonetheless high. Endovascular treatment cannot address any mass effect, and its therapeutic benefits are unclear; treatment of this type of aneurysm is very challenging and the prognosis is usually poor.

\section{Imaging follow-up strategies for intracranial arterial dissection Background}

The natural course and clinical outcomes of IAD are still unclear and mandate further investigation. Asymptomatic 
IADs are increasingly being detected as incidental findings on brain imaging. They may be much more common than previously thought, and the majority may arise and heal without any symptoms [6]. Nakagawa et al. found that the clinical course in most dissections is benign [10]. Vascular healing is normally complete within 2 months, but a minority of cases may be at risk of bleeding or rapid progression. Even if angiographic improvement of the dissection is observed, there is still a risk of SAH [54]. In the absence of strong evidence for the treatment of IAD, it is crucial to undertake clinical and imaging surveillance before intervention. The underlying pathophysiologic mechanisms of IAD are not the same as that of saccular aneurysms. Changes in the vessel lumen and wall should be assessed during follow-up, rather than simply focusing on luminal reconstruction. Some experts have recommended surgery only in patients with progressing symptomatic unruptured IAD. If symptoms remain stable, management should be conservative, with surveillance MRI performed 2 weeks, and 1, 3, 6, 12 and 24 months after onset. Once morphologic progression has been identified, surgery should be considered [55].

\section{Recommendations}

(1) Imaging follow-up of IAD should be carried out using standard methods tailored to the individual patient. Routine imaging review by MRI and MRA is required in the third and sixth month after presentation in cases managed conservatively. If clinical and imaging findings remain stable, MRI and MRA review should be undertaken annually. If new symptoms highly suspicious of dissection arise, imaging should be performed as soon as possible. (2) Patients with IAD may suffer from late recurrence and new bleeding. If endovascular treatment was successful, first imaging follow-up is recommended 3-6 months after treatment. First imaging reviews should be undertaken by MRI and MRA, or MRI and CTA, in patients with occlusion of the aneurysm and parent artery. As for patients who have undergone reconstructive treatment, DSA and MRI are recommended. Long-term follow-up by annual MRI and MRA is recommended in stable cases. Highresolution techniques can be used after treatment, as most modern clinical embolic materials (such as coils and intracranial stents) are paramagnetic. Although local artifact caused by metallic materials is inevitable, HR-MRI plays an indispensable role in identifying new IMH and signal changes within a lesion. Consequently, HR-MRI is recommended as the preferred non-invasive follow-up technique after endovascular treatment. Further examination by DSA is needed when follow-up findings are ambiguous.

\section{Conclusion}

This detailed document presents a Chinese specialist consensus on the imaging diagnosis of intracranial arterial dissection. Recommendations are made for clinicians to provide criteria for the diagnosis and classification of IADs. It also gives a comprehensive view of imaging features of IADs.

\section{Abbreviations}

3D-RA: Three-dimensional rotational angiography; CE-MRA: Contrastenhanced MRA; CTA: Computed tomography angiography; DSA: Digital subtraction angiography; HR-MRI: High-resolution MRI; IAD: Intracranial arterial dissection; IDA: Intracranial dissecting aneurysm; IMH: Intramural hematoma; MCA: Middle cerebral artery; MP-RAGE: Magnetization-prepared rapid gradient echo; MRA: Magnetic resonance angiography; MRI: Magnetic resonance imaging; PDWI: Proton density weighted images;

SAH: Subarachnoid hemorrhage; SNAP: Simultaneous non-contrast

angiography and intraplaque hemorrhage; SWI: Sensitivity-weighted imaging; T1WI: T1-Weighted imaging; T2WI: T2-Weighted imaging; TOF-MRA: Time of flight MRA

\section{Acknowledgements}

Not applicable.

Consensus Writing Committee (scheduled by Chinese phonetic alphabet):

Cai Jian-ming (Chinese PLA General Hospital),

Chen Zuo-quan (The Tenth Affiliated Hospital of Tongji Medical Sciences University),

Duan Chuan-zhi (Zhujiang Hospital, Southern Medical University),

Fan Yi-mu (Tianjin Huanhu Hospital),

Feng feng (Chinese Academy of Medical Sciences, Peking Union Medical College Hospital),

Gao Pei-yi (Beijing Tiantan Hospital affiliated to Capital Medical University), Gu Yu-xiang (Huashan Hospital Affiliated to Fudan University),

Guo You-min (The First Affiliated Hospital of Xi'an Jiao Tong University), Han Ping (Affiliated Union Hospital of Tongji Medical College,

Huazhong University of Science and Technology),

He Wei-wen (The Second Affiliated Hospital of Guangzhou Medical University),

Jia Wang (Beijing Tiantan Hospital affiliated to Capital Medical University),

Li Bao-min (Chinese PLA General Hospital),

Li Fei-yu (The First Hospital of Peking University),

Li You-xiang (Beijing Tiantan Hospital affiliated to Capital Medical University), Liang Chuan-sheng (The First Affiliated Hospital of China Medical University),

Liang Guo-biao (General Hospital of Shenyang Military Command),

Liu Jian-min (Changhai Hospital Affiliated to Second Military Medical University).

Liu Peng-fei (The First Affiliated Hospital of Harbin Medical University), Lu Guang-ming (Nanjing General Hospital of Nanjing Military Command), Luo Tian-you (The First Affiliated Hospital of Chongqing Medical University), Luo Qi (The First Bethune Hospital of Jilin University).

Lou Xin (Chinese PLA General Hospital),

Mao Geng-sheng (General Hospital of Chinese People's Armed Police ForcesMao Ying (Huashan Hospital Affiliated to Fudan University),

Miao Zhong-rong (Beijing Tiantan Hospital affiliated to Capital Medical University),

Shi Da-peng (Henan Province People's Hospital),

Shi Huai-zhang (The First Affiliated Hospital of Harbin Medical University),

Song Dong-lei (Donglei Brain Doctor Group),

Song Yan (Beijing Hospital of the Ministry of Health),

Sui Bin-bin (Beijing Tiantan Hospital affiliated to Capital Medical University),

Sun Yong-quan (Beijing Chaoyang Hospital affiliated to Capital Medical

University),

Wang Da-ming (Beijing Hospital of the Ministry of Health),

Wang Guang-bin (Shandong Medical Imaging Research Institute),

Wang Jun (Chinese PLA General Hospital),

Wang Shuo (Beijing Tiantan Hospital affiliated to Capital Medical University),

Wang Xiao-chun (The First Affiliated Hospital of Shanxi Medical University),

Wang Yang (The First Affiliated Hospital of Nanchang University),

Wu Zhong-xue (Beijing Tiantan Hospital affiliated to Capital Medical University),

Xing Xin-bo (Chinese PLA General Hospital),

Xu Kai (The Affiliated Hospital of Xuzhou Medical University),

Xue Yun-qing (Union Hospital Affiliated to Fujian Medical University), 
Yang Ming (Wuhan General Hospital of Guangzhou Military), Yang Xin-jian (Beijing Neurosurgical Institute),

Yin Long (Tianjin Huanhu Hospital),

Yu Jia-sheng (Affiliated Union Hospital of Tongji Medical College, Huazhong University of Science and Technology),

Yu Wei (Beijing Anzhen Hospital affiliated to Capital Medical University), Zhang Dong (Beijing Tiantan Hospital affiliated to Capital Medical University), Zhang Hui (The Affiliated Hospital of Shanxi Medical University),

Zhang Hong-qi (Capital Medical University Beijing Xuanwu Hospital), Zhang Xiao-long (Huashan Hospital Affiliated to Fudan University),

Zhao Xi-hai (Biomedical imaging research center of Tsinghua University), Zhao Zhen-wei (Tangdu Hospital of The Fourth Military Medical University), Zhong Ming (The Affiliated Hospital of Wenzhou Medical University).

\section{Funding}

This work was supported by National Natural Science Foundation of China (Grant Nos. 81,471,167 and 81,220,108,007), Special Research Project for Capita Health Development (Grant No.2014-1-.1071).

\section{Availability of data and materials}

All clinical data are stored by information system at Department of Interventional Neuroradiology, Beijing Neurosurgical Institute and Beijing Tian Tan Hospital, Capital Medical University.

\section{Authors' contributions}

YMW, YSZ, BBS, XJY and PYG performed the manuscript writing. All consensus writing committee members read and approved the final manuscript.

\section{Ethics approval and consent to participate}

Not applicable.

\section{Consent for publication}

Not applicable.

\section{Competing interests}

The authors declare that they have no competing interests.

\section{Author details}

'Department of Neurosurgery, First Affiliated Hospital of Zhengzhou University, Zhengzhou, Henan Province, China. ${ }^{2}$ Department of Interventional Neuroradiology, Beijing Neurosurgical Institute and Beijing Tian Tan Hospital, Capital Medical University, Beijing 100050, China. ${ }^{3}$ Department of Neuroimaging, Beijing Tiantan Hospital, Capital Medical University, Beijing 100050, China

Received: 13 April 2017 Accepted: 16 August 2017

\section{Published online: 02 November 2017}

\section{References}

1. Chineses Medical Association. Clinical diagnosis and treatment guidelines (cardiovascular surgery). People's medical publishing house. 2009;133-9.

2. Sikkema $\mathrm{T}$, Uyttenboogaart $\mathrm{M}$, Eshghi $\mathrm{O}$, et al. Intracranial artery dissection[J]. Eur J Neurol. 2014;21(6):820-6. doi:10.1111/ene.12384.

3. Debette S, Leys D. Cervical-artery dissections: predisposing factors, diagnosis, and outcome[J]. Lancet Neurol. 2009;8(7):668-78. doi:10.1016/ S1474-4422(09)70084-5.

4. Debette S, Compter A, Labeyrie MA, et al. Epidemiology, pathophysiology, diagnosis, and management of intracranial artery dissection[]]. Lancet Neurol. 2015;14(6):640-54. doi:10.1016/S1474-4422(15)00009-5.

5. Schievink WI. Spontaneous dissection of the carotid and vertebral arteries[]]. N Engl J Med. 2001;344(12):898-906. doi:10.1056/NEJM200103223441206.

6. Mizutani T. Natural course of intracranial arterial dissections[J]. J Neurosurg. 2011;114(4):1037-44. doi:10.3171/2010.9.JNS10668.

7. Han M, Rim NJ, Lee JS, et al. Feasibility of high-resolution MR imaging for the diagnosis of intracranial vertebrobasilar artery dissection[J]. Eur Radiol. 2014:24(12):3017-24. doi:10.1007/s00330-014-3296-5.

8. Nakatomi H, Segawa H, Kurata A, et al. Clinicopathological study of intracranial fusiform and dolichoectatic aneurysms : insight on the mechanism of growth[J]. Stroke. 2000;31(4):896-900.
9. Kim TW, Choi HS, Koo J, et al. Intramural hematoma detection by susceptibility-weighted imaging in intracranial vertebral artery dissection[J]. Cerebrovasc Dis. 2013;36(4):292-8. doi:10.1159/000354811.

10. Nakagawa $\mathrm{K}$, Touho H, Morisako T, et al. Long-term follow-up study of unruptured vertebral artery dissection: clinical outcomes and serial angiographic findings[J]. J Neurosurg. 2000;93(1):19-25. doi:10.3171/jns.2000. 93.1.0019.

11. Zhang $Y$, Wang $Y$, Sui $B$, et al. Magnetic resonance imaging follow-up of large or Giant Vertebrobasilar dissecting aneurysms after Total Embolization on angiography[]]. World Neurosurg. 2016;91:218-27. doi:10.1016/j.wneu.2016.04.024.

12. Nagahiro S, Takada A, Goto S, et al. Thrombosed growing giant aneurysms of the vertebral artery: growth mechanism and management[]]. J Neurosurg. 1995;82(5):796-801. doi:10.3171/jns.1995.82.5.0796.

13. Lin CH, Jeng JS, Yip PK. Middle cerebral artery dissections: differences between isolated and extended dissections of internal carotid artery[j]. J Neurol Sci. 2005;235(1-2):37-44. doi:10.1016/j.jns.2005.03.047.

14. Lee HO, Kwak HS, Chung GH, et al. Diagnostic usefulness of high resolution cross sectional MRI in symptomatic middle cerabral arterial dissection[J]. J Korean Neurosurg Soc. 2011:49(6):370-2. doi:10.3340/jkns.2011.49.6.370.

15. Provenzale JM, Sarikaya B. Comparison of test performance characteristics of MRI, MR angiography, and CT angiography in the diagnosis of carotid and vertebral artery dissection: a review of the medical literature[J]. AJR Am J Roentgenol. 2009;193(4):1167-74. doi:10.2214/AJR.08.1688.

16. Vertinsky AT, Schwartz NE, Fischbein NJ, et al. Comparison of multidetector CT angiography and MR imaging of cervical artery dissection[J]. AJNR Am J Neuroradiol. 2008;29(9):1753-60. doi:10.3174/ajnr.A1189.

17. Swartz RH, Bhuta SS, Farb Rl, et al. Intracranial arterial wall imaging using high-resolution 3-tesla contrast-enhanced MRI[]]. Neurology. 2009; 72(7):627-34. doi:10.1212/01.wnl.0000342470.69739.b3.

18. Bachmann R, Nassenstein I, Kooijman $\mathrm{H}$, et al. Spontaneous acute dissection of the internal carotid artery: high-resolution magnetic resonance imaging at 3.0 tesla with a dedicated surface coil[J]. Investig Radiol. 2006;41(2):105-11.

19. Naggara O, Louillet $F$, Touzé $E$, et al. Added value of high-resolution MR imaging in the diagnosis of vertebral artery dissection[J]. AJNR Am J Neuroradiol. 2010;31(9):1707-12. doi:10.3174/ajnr.A2165.

20. Wang Y, Lou X, Li Y, et al. Imaging investigation of intracranial arterial dissecting aneurysms by using 3 T high-resolution MRI and DSA: from the interventional neuroradiologists' view[J]. Acta Neurochir. 2014;156(3):515-25. doi:10.1007/s00701-013-1989-1.

21. Kwak HS, Hwang SB, Chung GH, et al. High-resolution magnetic resonance imaging of symptomatic middle cerebral artery dissection[]]. J Stroke Cerebrovasc Dis. 2014;23(3):550-3. doi:10.1016/j.jstrokecerebrovasdis.2013.03.019.

22. Ota H, Yarnykh VL, Ferguson MS, et al. Carotid intraplaque hemorrhage imaging at 3.0-T MR imaging: comparison of the diagnostic performance of three T1-weighted sequences[]]. Radiology. 2010;254(2):551-63. doi:10.1148/ radiol.09090535.

23. Wang J, Börnert $\mathrm{P}$, Zhao $\mathrm{H}$, et al. Simultaneous noncontrast angiography and intraplaque hemorrhage (SNAP) imaging for carotid atherosclerotic disease evaluation[J]. Magn Reson Med. 2013;69(2):337-45. doi:10.1002/mrm. 24254.

24. Hosoya T, Adachi M, Yamaguchi K, et al. Clinical and neuroradiological features of intracranial vertebrobasilar artery dissection[]]. Stroke. 1999;30(5): 1083-90.

25. Cuvinciuc V, Viallon M, Momjian-Mayor I, et al. 3D fat-saturated T1 SPACE sequence for the diagnosis of cervical artery dissection[J]. Neuroradiology. 2013;55(5):595-602. doi:10.1007/s00234-013-1141-1.

26. Edjlali M, Roca P, Rabrait C, et al. 3D fast spin-echo T1 black-blood imaging for the diagnosis of cervical artery dissection[J]. AJNR Am J Neuroradiol. 2013;34(9):E103-6. doi:10.3174/ajnr.A3261.

27. Takano K, Yamashita S, Takemoto K, et al. MRI of intracranial vertebral artery dissection: evaluation of intramural haematoma using a black blood, variable-flip-angle 3D turbo spin-echo sequence[J]. Neuroradiology. 2013; 55(7):845-51. doi:10.1007/s00234-013-1183-4.

28. Matsumoto S, Takada T, Yasaka M, et al. Intracranial arterial dissections in ischemic stroke assessed by 3D rotational angiography[]]. J Neurol Sci. 2010; 296(1-2):55-8. doi:10.1016/j.jns.2010.06.010.

29. Mokri B, Houser OW, Sandok BA, et al. Spontaneous dissections of the vertebral arteries[J]. Neurology. 1988;38(6):880-5.

30. Yoshimoto $Y$, Wakai $S$. Unruptured intracranial vertebral artery dissection. Clinical course and serial radiographic imagings[J]. Stroke. 1997;28(2):370-4 
31. Komotar RJ, Mocco J, Samuelson RM, et al. Rapidly successive, symptomatic, bilateral, spontaneous vertebral artery dissections: treatment with stent reconstruction[J]. Surg Neurol. 2009;72(3):300-5. doi:10.1016/j.surneu.2008.02.029.

32. Kim HS, Kwak HS, Hwang SB, et al. Rapid progression of symptomatic vertebrobasilar artery dissection on magnetic resonance imaging: a case report[J]. Neurointervention. 2014;9(2):106-8. doi:10.5469/neuroint.2014.9.2.106.

33. Asaithambi $G$, Saravanapavan $P$, Rastogi $V$, et al. Isolated middle cerebral artery dissection: a systematic review[J]. Int J Emerg Med. 2014;7(1):44. doi:10.1186/s12245-014-0044-1.

34. Houser OW, Mokri B, Sundt TM, et al. Spontaneous cervical cephalic arterial dissection and its residuum: angiographic spectrum[J]. AJNR Am J Neuroradiol. 1984;5(1):27-34

35. Klingebiel R, Busch $M$, Bohner $G$, et al. Multi-slice $C T$ angiography in the evaluation of patients with acute cerebrovascular disease-a promising new diagnostic tool[J]. J Neurol. 2002;249(1):43-9.

36. Ahmetoğlu K. Dinç H, et al. Multi-slice $\mathrm{CT}$ angiography in the diagnosis and characterization of cerebral aneurysms[]]. Tani Girisim Radyol. 2003;9(3):302-8.

37. Chen CJ, Tseng YC, Lee TH, et al. Multisection CT angiography compared with catheter angiography in diagnosing vertebral artery dissection[J]. AJNR Am J Neuroradiol. 2004;25(5):769-74.

38. Berne JD, Reuland KS, Villarreal DH, et al. Sixteen-slice multi-detector computed tomographic angiography improves the accuracy of screening for blunt cerebrovascular injury[J]. J Trauma. 2006;60(6):1204-1209; discussion 1209-1210. doi:10.1097/01.ta.0000220435.55791.ce.

39. Lum C, Chakraborty S, Schlossmacher M, et al. Vertebral artery dissection with a normal-appearing lumen at multisection $C T$ angiography: the importance of identifying wall hematoma[J]. AJNR Am J Neuroradiol. 2009; 30(4):787-92. doi:10.3174/ajnr.A1455.

40. Teasdale E, Zampakis P, Santosh C, et al. Multidetector computed tomography angiography: application in vertebral artery dissection[J]. Ann Indian Acad Neurol. 2011;14(1):35-41. doi:10.4103/0972-2327.78048.

41. Prabhakaran S, Romano JG. Current diagnosis and management of symptomatic intracranial atherosclerotic disease[J]. Curr Opin Neurol. 2012; 25(1):18-26. doi:10.1097/WCO.0b013e32834ec16b.

42. Wong LK. Global burden of intracranial atherosclerosis[J]. Int J Stroke. 2006; 1(3):158-9. doi:10.1111/j.1747-4949.2006.00045.x.

43. Ward MR, Pasterkamp G, Yeung AC, et al. Arterial remodeling. Mechanisms and clinical implications[J]. Circulation. 2000;102(10):1186-91.

44. Pasterkamp G, Wensing PJ, Post MJ, et al. Paradoxical arterial wall shrinkage may contribute to luminal narrowing of human atherosclerotic femoral arteries[J]. Circulation. 1995;91(5):1444-9.

45. Yuan C, Mitsumori LM, Beach KW, et al. Carotid atherosclerotic plaque: noninvasive MR characterization and identification of vulnerable lesions[J]. Radiology. 2001;221(2):285-99.

46. Wasserman BA, Wityk RJ, Trout HH 3rd, et al. Low-grade carotid stenosis: looking beyond the lumen with MRI[J]. Stroke. 2005;36(11):2504-13.

47. Koops A, Ittrich $\mathrm{H}$, Petri $\mathrm{S}$, et al. Multicontrast-weighted magnetic resonance imaging of atherosclerotic plaques at 3.0 and 1.5 Tesla: ex-vivo comparison with histopathologic correlation[J]. Eur Radiol. 2007;17(1):279-86.

48. Hunter MA, Santosh C, Teasdale E, et al. High-resolution double inversion recovery black-blood imaging of cervical artery dissection using 3T MR imaging[J]. AJNR Am J Neuroradiol. 2012;33(11):E133-7. doi:10.3174/ajnr.A2599.

49. Fatima Z, Motosugi U, Okumura A, et al. Basi-parallel anatomical scanning (BPAS)-MRI can improve discrimination of vertebral artery dissection from atherosclerosis and hypoplasia[J]. Acad Radiol. 2012;19(11):1362-7. doi:10. 1016/j.acra.2012.06.012

50. Mossa-Basha M, Hwang WD, De Havenon A, et al. Multicontrast highresolution vessel wall magnetic resonance imaging and its value in differentiating intracranial vasculopathic processes[J]. Stroke. 2015;46(6): 1567-73. doi:10.1161/STROKEAHA.115.009037.

51. Mizutani T, Miki Y, Kojima H, et al. Proposed classification of nonatherosclerotic cerebral fusiform and dissecting aneurysms[J]. Neurosurgery. 1999;45(2):253-9. discussion 259-260

52. Saliou G, Sacho RH, Power S, et al. Natural history and management of basilar trunk artery aneurysms[J]. Stroke. 2015;46(4):948-53. doi:10.1161/ STROKEAHA.114.006909.

53 Zhang Y, Tian Z, Sui B, et al. Endovascular treatment of spontaneous intracranial Fusiform and dissecting aneurysms: outcomes related to imaging classification of 309 cases. World Neurosurg. 2017;98:444-55.
54. Yoshikawa G, Kawamoto S, Ono H, et al. Vertebrobasilar artery dissection with subarachnoid hemorrhage after brain stem infarct showing an improvement on angiography: case report[]]. No Shinkei Geka. 2003;31(7):803-9.

55. Kai $Y$, Nishi $T$, Watanabe $M$, et al. Strategy for treating unruptured vertebral artery dissecting aneurysms[J]. Neurosurgery. 2011;69(5):1085-91; discussion 1091-1092. doi:10.1227/NEU.0b013e3182262adf.

\section{Submit your next manuscript to BioMed Central and we will help you at every step:}

- We accept pre-submission inquiries

- Our selector tool helps you to find the most relevant journal

- We provide round the clock customer support

- Convenient online submission

- Thorough peer review

- Inclusion in PubMed and all major indexing services

- Maximum visibility for your research

Submit your manuscript at www.biomedcentral.com/submit
Biomed Central 